\section{THE SUN AND THE IONOSPHERE}

$\mathrm{T}$ HE thirty-second Kelvin Lecture of the Institution of Electrical Engineers was delivered by Prof. Sydney Chapman on May 8, 1941, and has now been published ( $J$. Inst. Elect. Eng., 88, Pt. 1, No. 11, Nov., 1941). The first part of Prof. Chapman's lecture, dealing with the origin of solar energy by the 'combustion' of hydrogen, was printed in NATURE of June 28, 1941, p. 792 .

The process begins when the solar gas has reached a temperature of $20 \times 10^{6}{ }^{\circ} \mathrm{C}$. and a pressure of $10 \times 10^{9}$ atmospheres near the sun's centre, the 'burning' of the hydrogen nuclei thereafter maintaining the temperature and preventing further contraction for thousands of millions of years. In this reaction, the hydrogen protons are built up into helium nuclei during a six-stage reaction involving a catalyst, which at the outset is an ordinary carbon nucleus ${ }^{12} \mathrm{C}$. The equations of these reactions indicate a few million years for the fertilization of a carbon nucleus by four successive protons to beget one $\alpha$-particle. If the birth-rate is high at the sun's centre, it is on account of the immense number of carbon atoms and protons.

This catalytic reaction is enormously productive of energy, $1 \mathrm{lb}$. of hydrogen nuclei evolving radiant energy equivalent to 100 million electrical units, compared to $4 \frac{1}{2}$ units for $1 \mathrm{lb}$. of coal. Energy has a mass of 1 gm. per 25 million electrical units, and hence the radiant energy from I lb. of hydrogen nuclei has a mass of $4 \mathrm{gm}$., by which perceptible diminution the mass of the resulting helium nuclei falls short of $1 \mathrm{lb}$. For a loss in mass of four million tons/sec. the sun has a heating and lighting output of $10^{24} \mathrm{kw}$. Radiation leaves the sun with a spectral distribution corresponding roughly to $6,000^{\circ} \mathrm{C}$., consisting of highly directional electromagnetic waves, the average frequency of which is nearly a billion kc./sec. In the sun's upper layers the outward flowing radiation energizes many surface phenomena, the most evident being sunspots and prominences. Much solar gas in prominence form leaves the sun, which thus sends forth matter as well as radiation into surrounding space ; and this matter, in the form of rare gas streams, assists the solar radiation in the ionization of the earth's outer atmosphere.

All but a tiny fraction of the sun's colossal energy output is lost in space, the earth's input of 170 billion $\mathrm{kw}$. being but one thousand millionth of the parental output. Prior to entry into our atmosphere, this includes radiation of all wave-lengths, which may be resolved into a spectrum containing visible radiation or sunlight proper, ranging from violet to red (3900-7700 A.), and outside this the infra-red and ultra-violet. Much of the sun's light and heat pass through the atmosphere to the earth's surface, but the blue sunlight is dispersed. At an altitude of 15 miles above the atmosphere the sky is dark as at night. Energy absorbed by land and sea energizes almost all living processes, weather phenomena and geological changes, afterwards returning to space as heat radiation. Very little of the sun's radiation reaches the ground, the rest being absorbed at different levels by ozone, oxygen and nitrogen. Between 10 and 70 miles altitude, dissociated oxygen atoms combine with oxygen molecules to form ozone, an extremely rare constituent of the atmosphere, which, if collected at the earth's surface would, at the prevailing temperature, have a thickness less than $\frac{1}{8}$ in. This very small amount, spread through
30-40 miles of atmosphere, absorbs all the ultraviolet radiation (from about $2000 \mathrm{~A}$. to $3000 \mathrm{~A}$.), and also some of the visible and heat radiation-in all, about 5 per cent of the whole incident energy. At an altitude of 7 miles, the temperature is $220^{\circ}$ abs. $\left(-53^{\circ}\right.$ C. $)$, rising with increasing altitude to greater than $100^{\circ} \mathrm{C}$., cooling again after a further 25 miles, according to the band spectrum evidence of the Northern Lights.

The absorption of radiation in the extreme ultra. violet end of the spectrum can ionize molecular oxygen and nitrogen, which, absorbing at different levels, can provide a two-layer ionosphere. Appleton recognized the existence of two distinct main ionized layers : the lower or $E$ layer, at 60 miles, and the upper or $F$ layer. The latter in day-time in middle and lower latitudes consists of two parts, $F_{1}$ at 130 miles and $F_{2}$ at 200 miles. The $E$ layer appears to remain nearly constant in height, whereas the $F_{2}$ varies greatly. Absorption of the ionizing solar radiation is more likely to be due to molecules than to atoms. Molecular nitrogen extends upwards for hundreds of miles above the ground, whereas molecular oxygen is probably scarce at 70-80 miles; hence the ionized nitrogen layer is likely to be situated above the ionized oxygen layer and we may probably identify the nitrogen layer with $F$, and the oxygen layer with $E$. Appleton and Hulbert consider that the temperature of the $F_{2}$ layer rises, due to some radiant energy being absorbed and converted into heat within the layer, causing an upward expansion and rarefaction of the layer and demanding remarkably high temperatures of the order of $1,000^{\circ}$ abs. Such temperatures imply large random molecular velocities in the $F_{2}$ layer, weakening the earth's gravitational attraction on the air there, especially for hydrogen and helium, which probably leak away into space. Helium has constantly been reproduced radioactively from the earth's crust for more than a thousand million years, but the atmosphere does not contain the corresponding amount of helium. When the layer cools in the late afternoon it sinks, producing the abnormal second maximum of ionization.

Solar control of the ionosphere is indicated by the change in electron density following the 11-year sunspot cycle, the ionization having changed by 50-60 per cent between the sunspot minimum of 1934 and the sunspot maximum of 1937, implying a still greater increase, of 120-150 per cent, in the ultraviolet intensity. A similar change has long been revealed in the intensity of the system of electron currents flowing in the upper atmosphere, by their daily magnetic field variation, and this gave the first indication of an ionosphere, long before the advent of radio communication. The most remarkable irregular ionospheric changes are those associated with magnetic storms, causing long radio black-outs of hours or days and slowly rectifying abnormalities in the earth's magnetic field. The $F_{2}$ layer is most usually affected, except in high latitudes, where the $E$ region may be intensified and extended downwards to cause a radio black-out resembling those due to solar eruption, except that they may occur at night and be of longer duration. Appleton and his collaborators have discovered that the earth's field imposes a spiral character on the motion of electrons in the ionosphere, making the air anisotropic for the transmission of radio waves, permitting determination of the field intensity in the region of refraction of returned waves; and that the $E$ layer sustains lunar tidal motion twice per lunar day, the ionospheric 
tide having the unoxpected range of about 1 mile, and producing by dynamo action a system of electric currents, detectable by their magnetic effects at the ground, apparently flowing in or below the $E$ layer.

There also flows in the ionosphere a system of currents wholly governed by the sun, manifesting themselves by ordinary daily magnetic variations, the total current being about 200,000 amp., roughly. ten times as great as those due to lunar tides. The total current intensity in the greater system is only about twice that of a single lightning flash and the current density is extremely low, contributing inappreciably to the heating of the atmosphere. In a quite moderate storm there appears to be an intense current system (more than half a million amperes) in the ionosphere over each polar cap; the magnetic effects over the remaining 90 per cent of the earth betoken a current system of about the same total intensity.

\section{ELECTRONICS IN INDUSTRY}

$\mathrm{I}^{\mathrm{N}}$ May 1941, as a result of considerable interest in the various branches of industrial radiology, the Institute of Physics formed an Industrial Radiology Group (see Nature, June 7, 1941, p. 706). Recently there has been a widely expressed wish among members of the Institute for the formation of a similar group for electronics. It was decided, therefore, to hold a Conference on Electronics to ascertain whether there existed sufficient interest to justify the formation of such a group. This Conference was held at the Royal Institution on January 28 under the presidency of Prof. J. D. Cockeroft. It consisted in the reading of three papers followed by a general discussion and was well attended, members from many parts of Great Britain being present, as is usual at such meetings of the Institute.

Prof. Cockcroft, opening the proceedings, spoke of the widening field of application of electronic devices, and said that it is becoming increasingly difficult for physicists to keep in touch with developments in such specialist fields. He thought that the Conference would justify itself if it helped to minimize this difficulty.

The first paper, on "The Efficient Production of Light by the Electric Discharge", was read by V. J. Francis of the General Electric Company. The author pointed out that the scientific study of the efficient conversion of energy into radiation by means of electric discharges in gases is comparatively recent. Although the efficiency obtainable is several times that possible by any other known means, in practical lamps the efficiency of conversion is still only $12-15$ per cent, and a wide field for research still exists. Under certain ideal experimental conditions, efficiencies as great as 90 per cent have been recorded.

Mr. Francis then discussed the sodium and mercury vapour discharges in order to explain some of the principles involved in obtaining high luminous efficiency. The former requires low pressure and low current density because the resonance radiation is in a useful part of the visible spectrum; while with mercury, where the useful transitions are between excited states, the conditions leading to high efficiency are high pressure and high current density. He then dealt briefly with some of the applications of luminescent powders to discharge lamps. In one interesting application, practically all the light is obtained from the luminescent powder, which is excited by ultra- violet radiation produced with an efficiency of some 70 per cent in the low-pressure mercury vapour discharge. Finally, the possibilities of the mercury vapour discharge as a high-brightness source were mentioned; very high brightnesses are possible by this mears.

The next paper, on "The Control Characteristics of Thyratrons and Ignitrons", was given by H. de B. Knight, of the British Thomson-Houston Company. The author first dealt with the dependence of the ratings and control characteristics of hot-cathode thyratrons on cathode emission, on the geometry of the electrodes and envelope, and on the gas or vapour density. In gas-filled valves the density is constant and control characteristics are independent of temperature ; but the working voltage is limited to about 500 volts. For high power, mercury vapour is used ; but the vapour pressure must be controlled, especially for heavy current densities and high voltages (above $15 \mathrm{kv}$.) Mr. Knight showed many interesting curves illustrating these characteristics. Ionization time comprises a delay period in which practically no current passes, followed by a shorter 'build-up' period in which the plasma becomes established. Both periods are relatively independent of vapour pressure, but are affected by the grid voltage and resistance, and especially by the shapes of the electrodes and the field distribution in the are space. De-ionization time is a function not only of valve design but also of the circuit.

In dealing with mercury pool rectifiers, controlled by cathode spot formation, the author said that the 'Ignitron' alone finds wide industrial application. The delay time between the igniter current and the main arc depends on the rate of rise of igniter current. A delay of less than one microsecond can be obtained; but up to 50 microseconds or more is satisfactory for most industrial uses.

A. J. Maddock, of Standard Telephones and Cables, read the third paper, on "Hot-cathode Gas-filled Triodes (Thyratrons) and their Applications in Research and Industry". The author described the various ways in which these devices may be used. An outline of the principles of the several basie circuits that can be employed in most applications was given, and these were illustrated by interesting demonstrations so that the properties of the various circuits and the effects resulting from the use thereof were readily apparent.

The circuits discussed included those for use on A.c. and D.c. supplies, and showed the range of control obtainable in the former case as the point at which the grid loses control in each positive halfcycle of the alternating anode voltage is varied; and in the latter case how a second thyratron or switch may be used to stop the flow of current through a thyratron already passing current by applying a negative impulse to the anode of the first thyratron. Extension of this leads to the inverter circuit which produces an A.C. output from a D.C. input.

Several grid circuits were illustrated suitable for use in covering such applications and control as timing, impulse, variable reactance, variable resistance (including use of photo-electric cells), variable phase, etc. Examples of the use of these devices ranged from circuits for high-speed counting of physical phenomena and the generation of current pulses of accurate time duration used in research to large industrial plants such as high-tension rectifiers developing $600 \mathrm{kw}$. at 14,500 volts, large variablespeed motors operating on an A.c. supply, and 